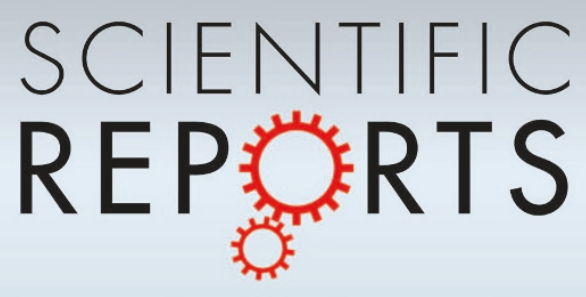

OPEN

SUBJECT AREAS:

CONDENSED MATTER

PHYSICS

ELECTRONIC PROPERTIES AND MATERIALS

SEMICONDUCTORS

SPINTRONICS

Received

15 January 2013

Accepted

18 March 2013

Published

11 April 2013

Correspondence and requests for materials should be addressed to W.Y. (wangyao@hku. hk) or X.D.C. (xdcui@ hku.hk)

\section{Optical signature of symmetry variations and spin-valley coupling in atomically thin tungsten dichalcogenides}

\author{
Hualing Zeng' ', Gui-Bin Liu' ${ }^{1,4,5}$, Junfeng Dai ${ }^{2,1}$, Yajun Yan ${ }^{3}$, Bairen Zhu' , Ruicong He', Lu Xie', Shijie Xu', \\ Xianhui Chen ${ }^{3}$, Wang Yao ${ }^{1,4}$ \& Xiaodong Cui ${ }^{1}$
}

'Department of Physics, The University of Hong Kong, Pokfulam road, Hong Kong, China, ${ }^{2}$ Department of Physics, South University of Science and Technology of China, Shenzhen, Guangdong, China, ${ }^{3}$ Hefei National Laboratory for physical Science at Microscale and Department of Physics, University of Science and Technology of China, Hefei, Anhui 230026, China, ${ }^{4}$ Center for Theoretical and Computational Physics, The University of Hong Kong, Hong Kong, China, ${ }^{5}$ School of Physics, Beijing Institute of Technology, Beijing 100081, China.

We report systematic optical studies of $\mathrm{WS}_{2}$ and WSe $\mathrm{W}_{2}$ monolayers and multilayers. The efficiency of second harmonic generation shows a dramatic even-odd oscillation with the number of layers, consistent with the presence (absence) of inversion symmetry in even-layer (odd-layer). Photoluminescence (PL) measurements show the crossover from an indirect band gap semiconductor at multilayers to a direct-gap one at monolayers. A hot luminescence peak $(\mathrm{B})$ is observed at $\sim 0.4 \mathrm{eV}$ above the prominent band edge peak (A) in all samples. The magnitude of A-B splitting is independent of the number of layers and coincides with the spin-valley coupling strength in monolayers. $A b$ initio calculations show that this thickness independent splitting pattern is a direct consequence of the giant spin-valley coupling which fully suppresses interlayer hopping at valence band edge near $\mathrm{K}$ points because of the sign change of the spin-valley coupling from layer to layer in the $2 \mathrm{H}$ stacking order.

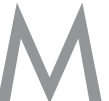

otivated by the triumph and limitation of graphene for electronic applications ${ }^{1}$, atomically thin layers of group VI transition metal dichalcogenides are attracting extensive interest as a class of graphene-like semiconductors with a desirable band-gap in the visible frequency range ${ }^{2-8}$. This family of dichalcogenides $\mathrm{MX}_{2}(\mathrm{M}=\mathrm{Mo}, \mathrm{W} ; \mathrm{X}=\mathrm{S}, \mathrm{Se})$ has a structure of $\mathrm{X}-\mathrm{M}-\mathrm{X}$ covalently bonded hexagonal quasi-2D network stacked by weak Van der Waals forces. $\mathrm{MX}_{2}$ thin films exhibit $2 \mathrm{H}$ stacking order: the neighboring layers are 180 degree in plane rotation of each other with the metal atom of a given layer sitting exactly on top of the chalcogenide atom of the adjacent layer. There is an even-odd variation in the structural symmetry of ultrathin films: inversion symmetry is absent (present) in films with odd (even) number of layers with space group of $\mathrm{D}^{1}{ }_{3 \mathrm{~h}}\left(\mathrm{D}^{4}{ }_{6 \mathrm{~h}}\right)$. Ab initio calculations predict that $\mathrm{MX}_{2}$ exhibits a transition from an indirect-gapped semiconductor in multilayer form to a direct bandgap one at visible range in monolayer, which has been experimentally verified in $\mathrm{MoS}_{2}{ }^{7,8}$.

$\mathrm{MX}_{2}$ monolayer, the elementary unit to form ultrathin films by weak stacking, features a novel spin-valley coupled band structure ${ }^{9}$. At the corners of the 1st Brillouin zone, the valence (conduction) band has two inequivalent valleys described by massive Dirac fermions. Owing to the broken inversion symmetry in monolayers, the strong spin-orbit coupling from the $\mathrm{d}$-orbitals of metal atom results in a valence band spin splitting at $\mathrm{K}$ points, with a magnitude as large as $\sim 0.4 \mathrm{eV}$ in tungsten dichalcogenides ${ }^{9,10}$. The spin-splitting has opposite signs at the $\mathrm{K}$ and $\mathrm{K}^{\prime}$ valleys as they are time reversal of each other. This spin-valley coupling forms the basis for manipulation of spin and valley degrees of freedom in these novel $2 \mathrm{D}$ semiconductors when combined with valley contrasted electric, magnetic and optical properties arising from inversion symmetry breaking ${ }^{9,11-16}$.

Here we report our experimental study on optical properties of ultrathin $\mathrm{WS}_{2}$ and $\mathrm{WSe}_{2}$ mono-, bi-, tri- and quad-layer samples by means of Raman scattering, second harmonic generation (SHG) and photoluminescence (PL). The efficiency of SHG at normal incidence on $\mathrm{WS}_{2}$ and $\mathrm{WSe}_{2}$ ultrathin films shows a dramatic even-odd oscillation with the number of layers: negligible at even-layer and nonzero at odd-layer, with maximum strength at monolayers. PL measurements demonstrate that $\mathrm{WS}_{2}$ and $\mathrm{WSe}_{2}$ exhibit a transition from an indirect-gap semiconductor at multi-layers to a direct-gap one at monolayers with an enhancement of the PL quantum efficiency (QE) at a factor of more than $10^{3}$ compared to bulk samples. Remarkably, a weak emission peak (B) is observed at an energy $\sim 0.4 \mathrm{eV}$ higher than the prominent direct bandgap transition peak (A) in all monolayer 
and multilayer samples. Unlike the case in $\mathrm{MoS}_{2}$ (B exicton absent in $\mathrm{MoS}_{2}$ monolayer PL though $)^{7}$, the magnitude of A-B splitting in both $\mathrm{WS}_{2}$ and $\mathrm{WSe}_{2}$ is independent of the number of layers and coincides with the spin-valley coupling strength in monolayers. Ab initio calculations show that this thickness independent splitting pattern is a direct consequence of the giant spin-valley coupling which fully suppresses interlayer hopping at valence band edge at $\mathrm{K}$ points because of the sign change of the spin-valley coupling from layer to layer in the $2 \mathrm{H}$ stacking order.

\section{Results}

For $\mathrm{MX}_{2}$ layered compounds, there are generally four Raman-active modes, namely $\mathrm{A}_{1 \mathrm{~g}}, \mathrm{E}_{1 \mathrm{~g}}, \mathrm{E}_{2 \mathrm{~g}}$ and $\mathrm{E}_{2 \mathrm{~g}}^{2}$ modes $^{17,18}$. $\mathrm{E}_{1 \mathrm{~g}}$ mode and low energy $E_{2 g}^{2}$ mode are absent in our measurements due to the forbidden selection rule in the back-scattering geometry and the limited rejection against Rayleigh scattering respectively. The presented study focuses on the in-plane vibrational $\mathrm{E}_{2 \mathrm{~g}}^{1}$ mode and the outof-plane vibrational $A_{1 g}$ mode. As these two modes are both polarization sensitive, the exciting laser line was tuned to an unpolarized state. Figure 2.a-d present the representative Raman spectra of $\mathrm{WS}_{2}$ and $\mathrm{WSe}_{2}$ slabs with layer number $\mathrm{N}=1$ to 4 and bulk. In the case of $\mathrm{WS}_{2}$, we observe the $\mathrm{E}_{2 \mathrm{~g}}^{1}$ mode at $\sim 350 \mathrm{~cm}^{-1}$ and the $\mathrm{A}_{1 \mathrm{~g}}$ mode at $\sim 420 \mathrm{~cm}^{-1}$ (Fig. 2.a) ${ }^{19}$. The $\mathrm{E}_{2 \mathrm{~g}}^{1}$ mode shows little dependence on the film thickness, while the $\mathrm{A}_{1 \mathrm{~g}}$ mode undergoes a blue shift with increasing layer number, showing a lattice stiffening effect as expected when additional layers are added. By examining the frequency differences $(\Delta \omega)$ between the $\mathrm{E}_{2 \mathrm{~g}}^{1}$ mode and $\mathrm{A}_{1 \mathrm{~g}}$ mode, the sample thickness could be identified accordingly. As indicated in Fig. 2.b showing the frequency difference as a function of layer number $\mathrm{N}$, we label $\Delta \omega=65.5 \mathrm{~cm}^{-1}, 68.3 \mathrm{~cm}^{-1}$ and $69.2 \mathrm{~cm}^{-1}$ to monolayer, bilayer and trilayer respectively. For slabs composed of four and more layers, $\Delta \omega$ converges to the bulk value at around $70 \mathrm{~cm}^{-1}$. Notably, from monolayer to trilayer the $A_{1 g}$ peak is roughly $0.5,1$ and 1.8 times the height of the $\mathrm{E}_{2 \mathrm{~g}}^{1}$ peak (Fig. 2.b), demonstrating that the ratio of the intensity of $\mathrm{A}_{1 \mathrm{~g}}$ mode to that of $\mathrm{E}_{2 \mathrm{~g}}^{1}$ mode could also be used as an indicator of sample thickness. For $\mathrm{WSe}_{2}$, two dominant peaks are observed around $250 \mathrm{~cm}^{-1}$ in various samples from monolayer to bulk (Fig. 2.c). However, little systematic trend could be observed on both the two modes as shown in Fig. 2.d.
An experimental method to examine the inversion symmetry in ultrathin film is to study the nonlinear optical effect such as SHG determined by the second order susceptibility $\chi^{(2)}{ }^{20}$. In the presence of inversion symmetry, $\chi^{(2)}$ is zero. A dramatic even-odd oscillation pattern is indeed observed on the SHG intensity consistent with the presence (absence) of inversion symmetry in even-layer (odd-layer) as shown in Fig. 1.c and 1.g. $\mathrm{WS}_{2}$ and $\mathrm{WSe}_{2}$ ultrathin slabs are scanned by a $150 \mathrm{fs}$ pulsed laser beam with a wavelength of $800 \mathrm{~nm}$ at normal incidence and the signal at the double frequency $(400 \mathrm{~nm})$ is collected. As expected, in both $\mathrm{WS}_{2}$ and WSe $\mathrm{W}_{2}$ slabs with even layer number or bulk samples, negligible SHG are observed as same in the case of the bare substrate $\left(\mathrm{SiO}_{2}\right.$ on $\left.\mathrm{Si}\right)$, and strong second harmonic emission arises from multilayer slabs with odd layer number. Notably, the brightest second harmonic emission is observed in monolayers of both $\mathrm{WS}_{2}$ and $\mathrm{WSe}_{2}$. The intensity of the second harmonic emission decays gradually with the increasing layer number, as indicated in Fig. 1.d and 1.h.

The Photoluminescence study shows that $\mathrm{WS}_{2}$ and $\mathrm{WSe}_{2}$ exhibit a transition from indirect band-gap semiconductor in the form of bulk and multilayers to direct band-gap one in monolayers, similar to $\mathrm{MoS}_{2}{ }^{7,8,21,22}$. Figure 3 illustrates the PL spectra of $\mathrm{WS}_{2}$ and $\mathrm{WSe}_{2}$ samples with various thicknesses measured under the same condition with an excitation at $2.41 \mathrm{eV}$. Fig. 3.a and 3.d show the PL peak intensity as a function of thickness. The PL intensity is found to be extremely weak on bulk samples, consistent with an indirect bandgap semiconductor in bulk form. As $\mathrm{WS}_{2}$ and $\mathrm{WSe}_{2}$ thin to a few atomic layers, the intensity of PL from direct interband transition dramatically increases and reaches maximum at monolayers, more than 3 orders of magnitude stronger than that from bulk. Both $\mathrm{WS}_{2}$ and $\mathrm{WSe}_{2}$ monolayers show much brighter PL with intensity at one order of magnitude higher than bilayers. The peak originating from the indirect band-gap transition (labeled as "I" in Fig. 3.b and 3.e) gradually shifts toward higher energy and fades to null at monolayers. These behaviors are fully consistent with the calculated band structures (see Fig. 4 and supplementary information).

Besides the peaks from indirect transition and the prominent direct transition peak (A), weak PL peak (B) is observed at higher energy in $\mathrm{WS}_{2}$ and $\mathrm{WSe}_{2}$ at all thickness. Note that the similar peak $\mathrm{B}$ appearing in $\mathrm{MoS}_{2}$ multilayers is absent in monolayers ${ }^{7}$. The linewidth of peak $\mathrm{A}$ and $\mathrm{B}$ implies their excitonic origin as the case in
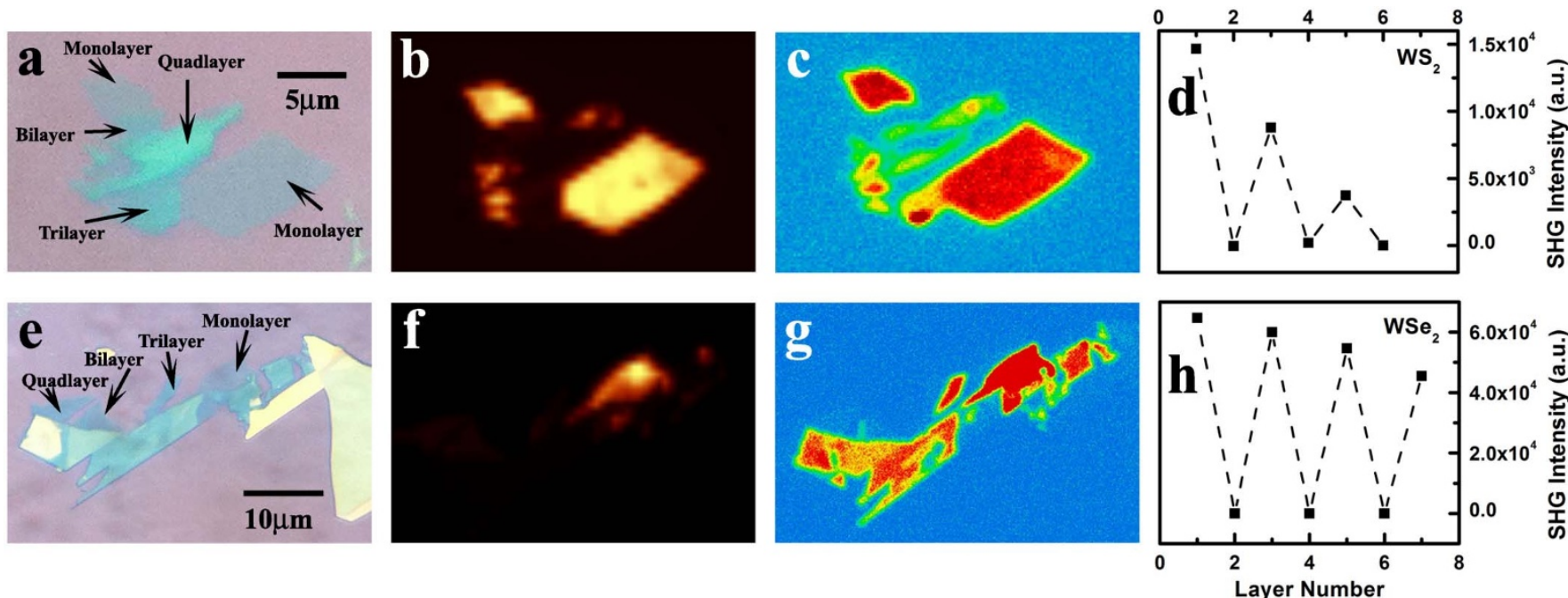

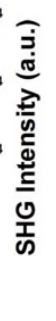

Figure $1 \mid$ (a) and (e): optical images of $\mathrm{WS}_{2}$ (a) and $\mathrm{WSe}_{2}$ (e) slabs on Si substrates with $300 \mathrm{~nm} \mathrm{SiO} \mathrm{S}_{2}$ cap-layer; (b) and (f): photoluminescence images at direct gap transition energy of the corresponding $\mathrm{WS}_{2}(\mathrm{~b})$ and $\mathrm{WSe}_{2}$ (f) slabs excited at $2.41 \mathrm{eV}$. Only monolayers are visible at the present contrast; (c) and (g): the corresponding SHG under a $800 \mathrm{~nm}$ excitation at normal incidence (150 fs, $80 \mathrm{MHZ}$ ) on WS $\mathrm{W}_{2}(\mathrm{c})$ and WSe 2 (g) respectively. The highest intensity labeled in red arises from monolayers. The SHG signal from the silicon/silicon oxide surface is negligible compared with those from multilayers; (d) and (h): The relative intensity of SHG as a function of the film thickness in $\mathrm{WS}_{2}(\mathrm{~d})$ and WSe 2 (h). The SHG shows an even-odd oscillation dependence on the layer number. 

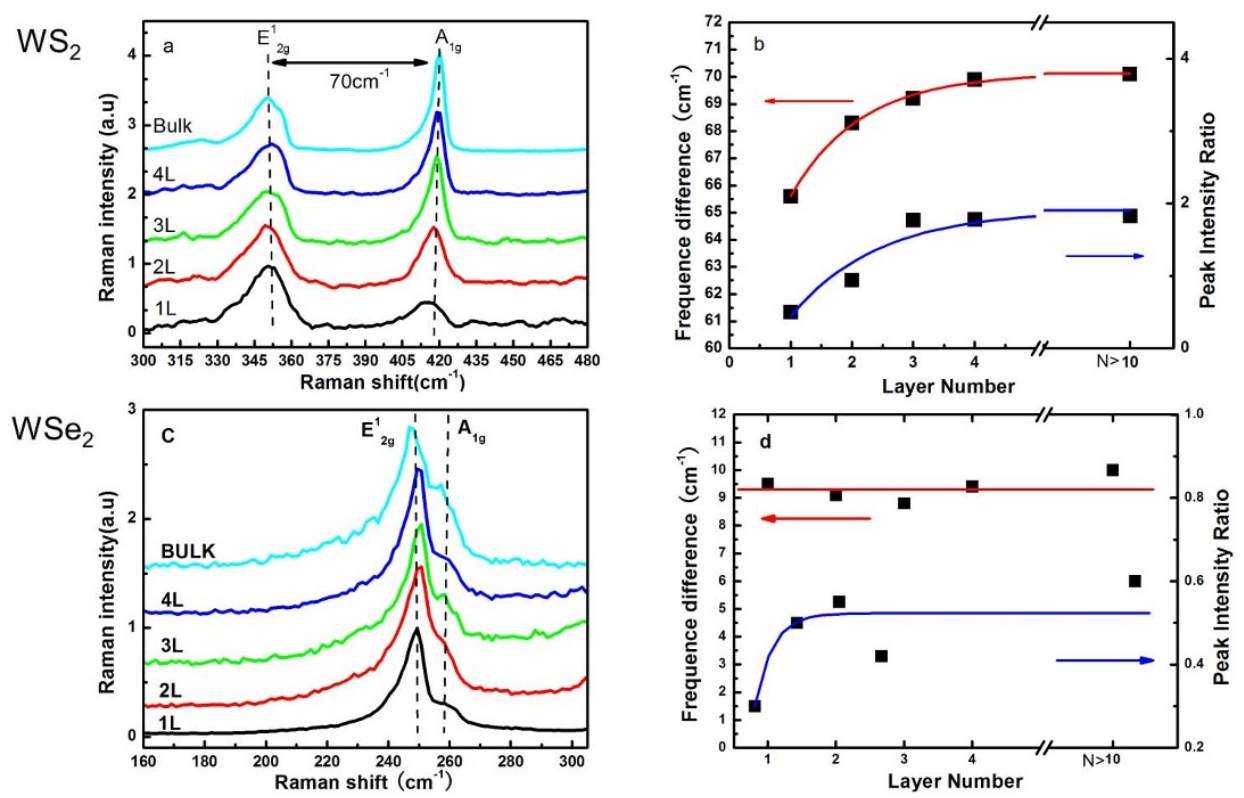

Figure $2 \mid$ (a) and (c): Raman spectra of $\mathrm{WS}_{2}$ (a) and $\mathrm{WSe}_{2}$ (c) ultrathin layers; (b) and (d): The frequency difference (red) and the peak intensity ratio (blue) between $\mathrm{E}_{2 \mathrm{~g}}^{1}$ and $\mathrm{A}_{1 \mathrm{~g}}$ modes as a function of film thickness in $\mathrm{WS}_{2}$ (b) and $\mathrm{WSe}_{2}$ (d) respectively.

$\mathrm{MoS}_{2}{ }^{7,23}$. Strikingly, the splitting between A and B peaks are almost identical, around $0.4 \mathrm{eV}$ for mono-, bi-, tri- and quad-layer samples (see Fig 3.c and 3.f). In monolayers, it is now well understood that the valence band edges at $\mathrm{K}$ points have a spin splitting purely arising from the strong spin-orbit coupling in the d-orbitals of the $\mathrm{W}$ atom, and we can attribute $A$ and $B$ to the direct-gap transitions between the spin split valence bands and the conduction band at the K points ${ }^{7}$. However, in multilayers, both the spin-orbit coupling and the interlayer hopping contribute to the valence band splitting at $\mathrm{K}$ points. Besides, even layer samples are inversion symmetric while odd layer samples are asymmetric. These in general would result in complex and layer-number dependent splitting patterns in multilayers, which
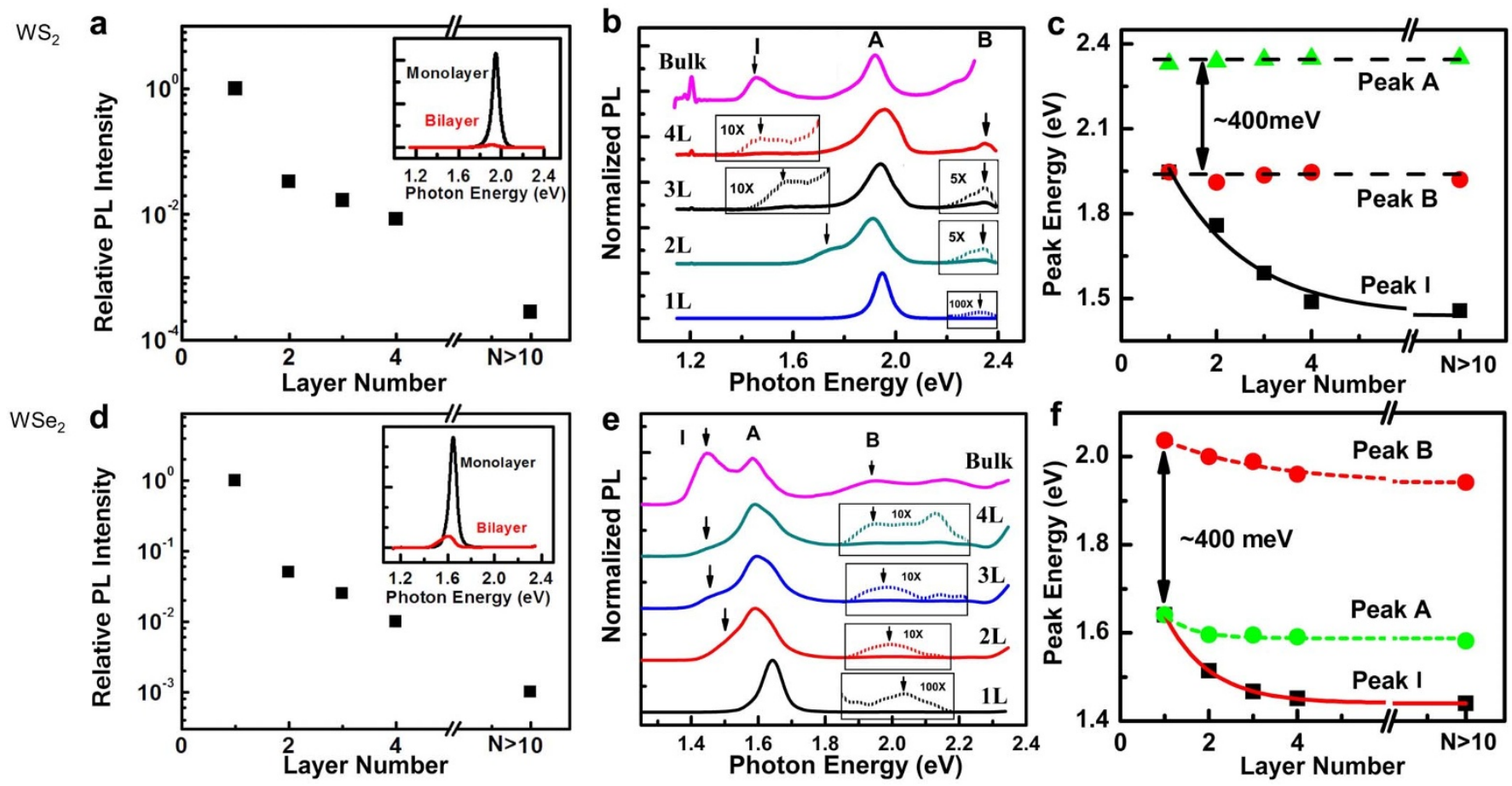

Figure $3 \mid$ (a) and (d): The relative PL intensity of $\mathrm{WS}_{2}(\mathrm{a})$ and $\mathrm{WSe}_{2}(\mathrm{~d})$ multilayers respectively as a function of film thickness under the same conditions (normalized by the PL intensity of monolayer at 1). Insets present PL spectra from $\mathrm{WS}_{2}$ (a) and $\mathrm{WSe}_{2}(\mathrm{~d})$ monolayers and bilayers respectively. The spectra were taken at the same conditions (excitation power, exposure time, etc.); (b) and (e): The normalized PL spectra (with respect to the peak A) of WS (b) $^{2}$ and $\mathrm{WSe}_{2}$ (e) ultrathin films. I labels the luminescence from indirect gap interband transition, A and B label the direct-gap transitions from the split valence band edge to the conduction band edge at $\mathrm{K}$ points (see text). Spectra (dash line) in the zoom windows have been multiplied by a factor as indicated for clarity; (c) and (f): The peak positions of I, A and B transitions as a function of the film thickness in WS $\mathrm{W}_{2}(\mathrm{c})$ and WSe $\mathrm{B}_{2}$ (f). Both cases show a nearly constant energy difference of $\sim 0.4 \mathrm{eV}$ which corresponds to the splitting of the valence band edge. The universal A-B splitting implies a suppression of interlayer hopping in tungsten dichalcogenides ultrathin thin films. 


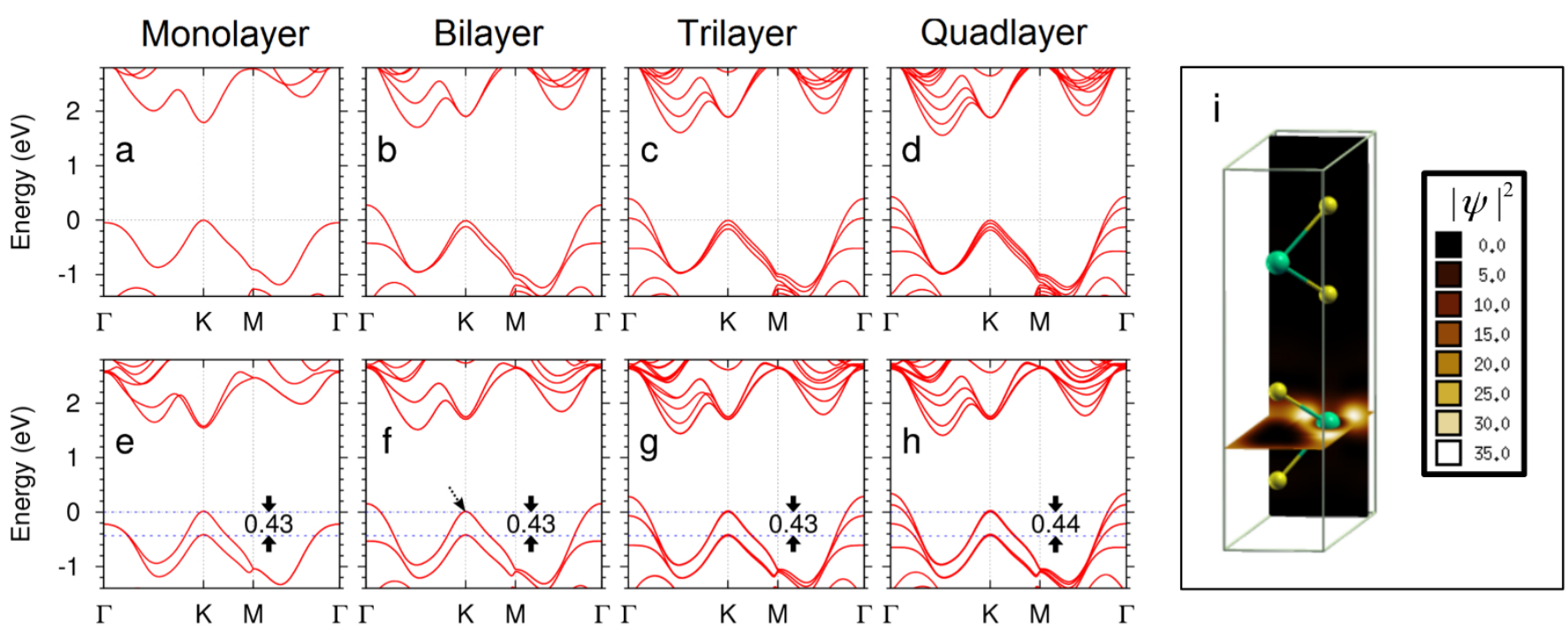

Figure $4 \mid$ Ab initio calculated band structures for $\mathrm{WS}_{2}$ ultrathin films. (a-d): without spin-orbit coupling; (e-h): with spin-orbit coupling. The valence band splittings at $\mathrm{K}$ point are nearly constants, around $0.43 \mathrm{eV}$ for mono-,bi-, tri, and quad-layers; (i) Electron density map for one valence band Bloch state at $\mathrm{K}$ point in $\mathrm{WS}_{2}$ bilayer (indicated by the dashed arrow in (f)). The wavefunction is fully spin polarized in the down state and almost fully localized in the bottom layer. The other degenerate state at the same K point can be obtained by a spatial inversion plus a time reversal operation.

is obviously different from the universal A-B splitting in the PL spectra observed. It implies a novel cause in tungsten dichalcogenides ultrathin thin films.

\section{Discussion}

To understand the A-B splitting pattern in $\mathrm{WS}_{2}$ and $\mathrm{WSe}_{2}$ ultrathin films, we perform ab initio calculations of the band structures using the projector augmented wave method ${ }^{24}$ and generalized gradient approximation $^{25}$ implemented in the ABINIT code ${ }^{26,27}$. The structure parameters are taken from Ref. 10. Figure 4.a-d show the band structures of mono-, bi-, tri-, and quad-layer $\mathrm{WS}_{2}$ in the absence of SOC. As expected, the valence band edge at $\mathrm{K}$ point splits into two, three, and four bands respectively for bi-, tri-, and quad-layer $\mathrm{WS}_{2}$ due to the interlayer hopping. A hopping matrix element $\mathrm{t} \sim 0.1 \mathrm{eV}$ can be extracted from the splitting pattern. However, when spinorbit coupling (SOC) is included, the splitting pattern is completely changed as shown in Fig. 4.e-h. The valence band edges split into two degenerate manifolds with a splitting magnitude independent of the film thickness for both $\mathrm{WS}_{2}$ and $\mathrm{WSe}_{2}$. The band structures of $\mathrm{WSe}_{2}$ ultrathin films could be found in supplementary information. This is in perfect agreement with the A-B splitting patterns observed in the photoluminescence of mono-, bi-, tri-, and quad-layer $\mathrm{WS}_{2}$ and $\mathrm{WSe}_{2}$. The calculated valence band edge splittings of $0.43 \mathrm{eV}$ in $\mathrm{WS}_{2}$ and $0.47 \mathrm{eV}$ in $\mathrm{WSe}_{2}$ also agree with the measured A-B splitting of $0.4 \mathrm{eV}$ as shown in Fig. 3 and the supplementary information.

In fact, the unexpected splitting patterns in multilayer $\mathrm{WS}_{2}$ and $\mathrm{WSe}_{2}$ are manifestations of the giant spin-valley coupling in valence band ${ }^{9}$. In monolayers, the Kramer's doublet $\left|K^{\uparrow}\right\rangle$ and $\left|K^{\prime} \downarrow\right\rangle$ are separated from the other doublet $\left|K^{\prime} \uparrow\right\rangle$ and $|K \downarrow\rangle$ by the spin-valley coupling energy of $\lambda_{s v c} \sim 0.4 \mathrm{eV}^{9}$. In the $2 \mathrm{H}$ stacked multilayers, any two neighboring layers are 180 degree in plane rotation of each other. This rotation switches $\mathrm{K}$ and $\mathrm{K}^{\prime}$ valleys but leaves the spin unchanged, which results in a sign change for the spin-valley coupling from layer to layer. Thus, the spin-conserving interlayer hopping can only couple states in neighboring layers with a detuning $\lambda_{s v c}$. Interlayer hopping is therefore strongly suppressed by the giant spin-valley coupling. A direct consequence is that the splitting patterns remain the same as that of monolayers, and the valence band Bloch states near $\mathrm{K}$ points are largely localized in individual layers, as if the interlayer hopping is absent. This is indeed confirmed by ab initio calculations of electron density distributions of these Bloch states (see Fig. 4.i and table S2 in supplementary information). The full suppression of interlayer hopping at $\mathrm{K}$ points by the spin-valley coupling is unique to tungsten dichalcogenides where $\lambda_{s v c} \gg t$. For molybdenum dichalcogenides where $\lambda_{s v c} \sim t$, interlayer hopping will manifest in both the splitting pattern and Bloch function ${ }^{28}$.

The above picture also explains why the hot luminescence peak B can be observable from monolayers to multilayers at an energy much higher than the prominent direct bandgap transition peak A. In monolayers, near $\mathrm{K}$ points, the spin-valley coupling gives rise to two non-degenerate valence bands with opposite spin separated by an energy of $\lambda_{s v c} \sim 0.4 \mathrm{eV}$. The relaxation of hot carriers between the spin-split valence bands requires a spin flip and is much slower than intra-band relaxation. Hot carriers can thus have a finite lifetime at the higher energy band edge, giving rise to hot luminescence, i.e. the direct bandgap transition peak $\mathrm{B}^{8}$. In multilayers, we have the same picture for hot carrier relaxation and hot luminescence at the valence band edges near $\mathrm{K}$ points where interlayer hopping is fully suppressed by the spin-valley coupling. The giant spin-valley coupling offers a new strategy to manipulate electron spin and makes tungsten dichalcogenides ultrathin films promising materials for semiconductor based spintronics and conceptual valley-based electronics.

\section{Methods}

$\mathrm{WS}_{2}$ and $\mathrm{WSe}_{2}$ flakes were mechanically exfoliated from synthesized single crystal bulk samples onto silicon wafers capped with a $300 \mathrm{~nm}$ thick $\mathrm{SiO}_{2}$ by a method analogous to the way of producing graphene ${ }^{1}$. $\mathrm{WS}_{2}$ and $\mathrm{WSe}_{2}$ slabs were first visually screened with interference color through optical microscope. Typical optical images of $\mathrm{WS}_{2}$ and $\mathrm{WSe}_{2}$ ultrathin slabs are presented in Fig. 1.a and 1.e. The film thickness is confirmed by atomic force microscope. PL spectra are also used as an indicator of monolayer samples (Fig. 1.b and 1.f). Raman scattering was carried out with a confocal setup.

1. Novoselov, K. S. et al. Electric Field Effect in Atomically Thin Carbon Films. Science 306, 666-669 (2004).

2. Novoselov, K. S. et al. Two-dimensional atomic crystals. Proceedings of the National Academy of Sciences of the United States of America 102, 10451-10453 (2005).

3. Coleman, J. N. et al. Two-Dimensional Nanosheets Produced by Liquid Exfoliation of Layered Materials. Science 331, 568-571 (2011).

4. Podzorov, V., Gershenson, M. E., Kloc, C., Zeis, R. \& Bucher, E. High-mobility field-effect transistors based on transition metal dichalcogenides. Applied Physics Letters 84, 3301-3303 (2004).

5. Radisavljevic, B., Radenovic, A., Brivio, J., Giacometti, V. \& Kis, A. Single-layer $\mathrm{MoS}_{2}$ transistors. Nat Nano 6, 147-150 (2011). 
6. Fang, H. et al. High-Performance Single Layered WSe $\mathrm{W}_{2}$ p-FETs with Chemically Doped Contacts. Nano Letters 12, 3788-3792 (2012).

7. Mak, K. F., Lee, C., Hone, J., Shan, J. \& Heinz, T. F. Atomically Thin $\mathrm{MoS}_{2}$ : A New Direct-Gap Semiconductor. Physical Review Letters 105, 136805 (2010).

8. Splendiani, A. et al. Emerging Photoluminescence in Monolayer $\mathrm{MoS}_{2}$. Nano Letters 10, 1271-1275 (2010)

9. Xiao, D., Liu, G.-B., Feng, W., Xu, X. \& Yao, W. Coupled Spin and Valley Physics in Monolayers of $\mathrm{MoS}_{2}$ and Other Group-VI Dichalcogenides. Physical Review Letters 108, 196802 (2012).

10. Zhu, Z. Y., Cheng, Y. C. \& Schwingenschlögl, U. Giant spin-orbit-induced spin splitting in two-dimensional transition-metal dichalcogenide semiconductors. Physical Review B 84, 153402 (2011).

11. Xiao, D., Yao, W. \& Niu, Q. Valley-Contrasting Physics in Graphene: Magnetic Moment and Topological Transport. Physical Review Letters 99, 236809 (2007).

12. Yao, W., Xiao, D. \& Niu, Q. Valley-dependent optoelectronics from inversion symmetry breaking. Physical Review B 77, 235406 (2008).

13. Cao, T. et al. Valley-selective circular dichroism of monolayer molybdenum disulphide. Nat Commun 3, 887 (2012).

14. Zeng, H., Dai, J., Yao, W., Xiao, D. \& Cui, X. Valley polarization in $\mathrm{MoS}_{2}$ monolayers by optical pumping. Nat Nano 7, 490-493 (2012).

15. Mak, K. F., He, K., Shan, J. \& Heinz, T. F. Control of valley polarization in monolayer $\mathrm{MoS}_{2}$ by optical helicity. Nat Nano 7, 494-498 (2012).

16. Sallen, G. et al. Robust optical emission polarization in $\mathrm{MoS}_{2}$ monolayers through selective valley excitation. Physical Review B 86, 081301 (2012).

17. Verble, J. L. \& Wieting, T. J. Lattice Mode Degeneracy in $\mathrm{MoS}_{2}$ and Other Layer Compounds. Physical Review Letters 25, 362-365 (1970).

18. Molina-Sánchez, A. \& Wirtz, L. Phonons in single-layer and few-layer $\mathrm{MoS}_{2}$ and $\mathrm{WS}_{2}$. Physical Review B 84, 155413 (2011).

19. Ramakrishna Matte, H. S. S. et al. $\mathrm{MoS}_{2}$ and $\mathrm{WS}_{2}$ Analogues of Graphene. Angewandte Chemie International Edition 49, 4059-4062 (2010).

20. Shen, Y. R. Principles of nonlinear optics. (1984).

21. Li, T. \& Galli, G. Electronic Properties of $\mathrm{MoS}_{2}$ Nanoparticles. The Journal of Physical Chemistry C 111, 16192-16196 (2007)

22. Lebègue, S. \& Eriksson, O. Electronic structure of two-dimensional crystals from ab initio theory. Physical Review B 79, 115409 (2009).

23. Korn, T., Heydrich, S., Hirmer, M., Schmutzler, J. \& Schuller, C. Low-temperature photocarrier dynamics in monolayer $\mathrm{MoS}_{2}$. Applied Physics Letters 99, 102109 (2011)

24. Torrent, M., Jollet, F., Bottin, F., Zérah, G. \& Gonze, X. Implementation of the projector augmented-wave method in the ABINIT code: Application to the study of iron under pressure. Computational Materials Science 42, 337-351 (2008).
25. Perdew, J. P., Burke, K. \& Ernzerhof, M. Generalized Gradient Approximation Made Simple. Physical Review Letters 77, 3865-3868 (1996).

26. Gonze, X. A brief introduction to the ABINIT software package. Zeitschrift für Kristallographie 220, 558-562 (2005).

27. Gonze, X. et al. First-principles computation of material properties: the ABINIT software project. Computational Materials Science 25, 478-492 (2002).

28. Cheiwchanchamnangij, T. \& Lambrecht, W. R. L. Quasiparticle band structure calculation of monolayer, bilayer, and bulk $\mathrm{MoS}_{2}$. Physical Review B 85, 205302 (2012)

\section{Acknowledgements}

We thank Mr. Xiaohu Wang, Dr. Changcheng Zheng and Dr. Helen Leung for technique assistance. J.D. acknowledges the financial support from National Natural Science Foundation of China (\#11204184). The work was supported by University Grant Council (AoE/P-04/08) and the Research Grant Council (HKU706412P) of the government of Hong Kong SAR and the National Basic Research Program of China (973 Program, Grant No. 2012CB922002)

\section{Author contributions}

X.C. and H.Z. designed the experiments. X.Chen. and Y.Y. fabricated the single crystal $\mathrm{WS}_{2}$ and $\mathrm{WSe}_{2}$, H.Z., J.D., B.Z., R.H. and L.X. performed the experiments. W.Y. and G.L. contributed to the theoretical interpretations and ab initio calculations. All authors discussed the results and co-wrote the paper.

\section{Additional information}

Supplementary information accompanies this paper at http://www.nature.com/ scientificreports

Competing financial interests: The authors declare no competing financial interests.

License: This work is licensed under a Creative Commons

Attribution-NonCommercial-NoDerivs 3.0 Unported License. To view a copy of this license, visit http://creativecommons.org/licenses/by-nc-nd/3.0/

How to cite this article: Zeng, H. et al. Optical signature of symmetry variations and spin-valley coupling in atomically thin tungsten dichalcogenides. Sci. Rep. 3, 1608; DOI:10.1038/srep01608 (2013). 